\title{
THE RIESZ KERNELS DO NOT GIVE RISE TO HIGHER DIMENSIONAL ANALOGUES OF THE MENGER-MELNIKOV CURVATURE
}

\author{
HANY M. FARAG
}

\begin{abstract}
Ever since the discovery of the connection between the MengerMelnikov curvature and the Cauchy kernel in the $L^{2}$ norm, and its impressive utility in the analytic capacity problem, higher dimensional analogues have been coveted. The lesson from 1-sets was that any such (nontrivial, nonnegative) expression, using the Riesz kernels for $m$-sets in $\mathbf{R}^{n}$, even in any $L^{k}$ norm $(k \in \mathbf{N})$, would probably carry nontrivial information on whether the boundedness of these kernels in the appropriate norm implies rectifiability properties of the underlying sets or measures. Answering such questions would also have an impact on another important problem, namely whether totally unrectifiable $m$-sets are removable for Lipschitz harmonic functions in $\mathbf{R}^{m+1}$. It has generally been believed that some such expressions should exist at least for some choices of $m, k$, or $n$, but the apparent complexity involved made the search rather difficult, even with the aid of computers. However, our rather surprising result is that, in fact, not a single higher dimensional analogue of this useful curvature can be derived from the Riesz kernels in the same fashion, and that, even for 1-sets, the Menger-Melnikov curvature is unique in a certain sense.
\end{abstract}

\section{Introduction}

Recall that the so-called Menger-Melnikov curvature $c(x, y, z)$ is defined to be the reciprocal of the radius of the circle passing through the (distinct) points $x, y, z ; c(x, y, z)=0$ if and only if $x, y, z$ are collinear. Melnikov $[\mathbf{M e}]$ found the identity (for distinct $z_{1}, z_{2}, z_{3} \in \mathbf{C}$ ):

$$
c\left(z_{1}, z_{2}, z_{3}\right)^{2}=\sum_{\sigma} \frac{1}{\left(z_{\sigma(1)}-z_{\sigma(3)}\right) \overline{\left(z_{\sigma(2)}-z_{\sigma(3)}\right)}},
$$


where the sum is over all six permutations $\sigma$ of $\{1,2,3\}$. The same identity can be transformed into (for distinct $x_{1}, x_{2}, x_{3} \in \mathbf{R}^{n}$ ):

$$
c\left(x_{1}, x_{2}, x_{3}\right)^{2}=\sum_{\sigma} \frac{\left(x_{\sigma(1)}-x_{\sigma(3)}\right) \cdot\left(x_{\sigma(2)}-x_{\sigma(3)}\right)}{\left|x_{\sigma(1)}-x_{\sigma(3)}\right|^{2}\left|x_{\sigma(2)}-x_{\sigma(3)}\right|^{2}} .
$$

It is also a simple exercise to show that

$$
c\left(x_{1}, x_{2}, x_{3}\right)^{2}=4\left\{\frac{\left|x_{1}-x_{3}\right|^{2}\left|x_{2}-x_{3}\right|^{2}-\left(\left(x_{1}-x_{3}\right) \cdot\left(x_{2}-x_{3}\right)\right)^{2}}{\left|x_{1}-x_{3}\right|^{2}\left|x_{2}-x_{3}\right|^{2}\left|x_{1}-x_{2}\right|^{2}}\right\},
$$

which, by the Schwarz inequality, shows explicitly the nonnegativity of this expression. Now suppose $\mu$ is a finite Borel measure, and that $\int \frac{\left(x_{1}-x_{3}\right)}{\left|x_{1}-x_{3}\right|^{2}} d \mu\left(x_{1}\right)$ is in $L^{2}(\mu)$. Then (ignoring any subtleties), we might formally argue that

(4)

$$
\begin{aligned}
& \frac{1}{6} \iiint c\left(x_{1}, x_{2}, x_{3}\right)^{2} d \mu\left(x_{1}\right) d \mu\left(x_{2}\right) d \mu\left(x_{3}\right) \\
= & \frac{1}{6} \iiint \sum_{\sigma} \frac{\left(x_{\sigma(1)}-x_{\sigma(3)}\right)}{\left|x_{\sigma(1)}-x_{\sigma(3)}\right|^{2}} \cdot \frac{\left(x_{\sigma(2)}-x_{\sigma(3)}\right)}{\left|x_{\sigma(2)}-x_{\sigma(3)}\right|^{2}} d \mu\left(x_{\sigma(1)}\right) d \mu\left(x_{\sigma(2)}\right) d \mu\left(x_{\sigma(3)}\right) \\
= & \frac{1}{6} \sum_{\sigma} \iiint \frac{\left(x_{\sigma(1)}-x_{\sigma(3)}\right)}{\left|x_{\sigma(1)}-x_{\sigma(3)}\right|^{2}} \cdot \frac{\left(x_{\sigma(2)}-x_{\sigma(3)}\right)}{\left|x_{\sigma(2)}-x_{\sigma(3)}\right|^{2}} d \mu\left(x_{\sigma(1)}\right) d \mu\left(x_{\sigma(2)}\right) d \mu\left(x_{\sigma(3)}\right) \\
= & \iiint \frac{\left(x_{1}-x_{3}\right)}{\left|x_{1}-x_{3}\right|^{2}} \cdot \frac{\left(x_{2}-x_{3}\right)}{\left|x_{2}-x_{3}\right|^{2}} d \mu\left(x_{1}\right) d \mu\left(x_{2}\right) d \mu\left(x_{3}\right)<\infty .
\end{aligned}
$$

This is not quite true of course, but see e.g. [MMV] where the difficulties are handled (at least for appropriate types of measures). In particular, if $\mu$ is the restriction of the one dimensional Hausdorff measure to an A-D regular set it is proved using techniques developed in $[\mathbf{J o}],[\mathbf{D S 1}]$, and [DS2] that the set is uniformly rectifiable. Recall that a set $E$ is said to be an A-D regular 1-set if there exists a constant $C<\infty$ such that

$$
C^{-1} r \leq H^{1}(E \cap B(x, r)) \leq C r
$$

whenever $x \in E$, and $0<r<\operatorname{diam}(E)$. Combining this with a theorem of Michael Christ $[\mathbf{C h}]$, the authors prove that, if an A-D 1-set has positive analytic capacity, then it must be uniformly rectifiable. Also, Melnikov and Verdera $[\mathbf{M V}]$ gave a geometric proof that the Cauchy integral is bounded in $L^{2}$ on Lipschitz graphs, using this curvature. 
All these developments finally led to the solution of the analytic capacity problem [Da] (namely that compact totally unrectifiable 1-sets in the plane are removable for bounded analytic functions). One is then interested in doing the same for $m$ dimensional sets $(m \geq 2)$ in $\mathbf{R}^{n}$. For these sets the natural analogues with the right scaling are the Riesz kernels $K_{m}=x|x|^{-m-1}$. These kernels, among others, were investigated in $[\mathbf{D S 1}],[\mathbf{D S 2}]$ on A-D regular sets, and the question of whether $\left.K_{m} * d \mathcal{H}^{1}\right|_{E} \in L^{2}(E)$ implies uniform rectifiability of the set is still open for $m \geq 2$. One would have hoped that a similar approach might work, perhaps using an $L^{k}$ norm, with a $k$ that may depend on $m$ (or even $n$ if necessary). Another very interesting open question of a similar nature to the analytic capacity problem is whether totally unrectifiable sets are removable for Lipschitz harmonic functions. There, the only relevant sets are $m$-sets in $\mathbf{R}^{m+1}$, with $m \geq 2$ (for $m=1$ this was resolved in $[\mathbf{D M}]$, also making use of the Menger-Melnikov curvature). See also $[\mathbf{M P}]$ where it is proved that a wide subclass of these sets are removable. It therefore became of great interest to investigate expressions of the form (2), but with the power 2 in the denominator replaced by $m+1$, and also allowing more points (i.e. allowing $L^{k}$ norms with $k \geq 2$ ). The results of the next section show that we are fortunate to have the Menger-Melnikov curvature for 1-sets, because for $m \geq 2$ there are no analogues of it that can be derived from the Riesz kernels in the same fashion or, in other words, there is no identity similar to that of Melnikov's relating certain norms of these kernels to some (nontrivial, and nonnegative) expression.

I would like to thank Stephen Semmes for useful conversations regarding this work, and Joan Verdera for valuable comments.

\section{The Main Theorem}

\subsection{Definitions.}

For the rest of the paper we will assume that $m \geq 1, k \geq 1, n \geq 2$ are integers (note that rectifiability theory is trivial for $n=1$ ). We let $\mathcal{P}$ be the class of all functions of the form

$$
\begin{aligned}
& P_{m, k, n}\left(x_{0}, x_{1}, \ldots, x_{k}\right) \\
& =\sum_{\gamma \in H} \sum_{j_{1}=1}^{n} \cdots \sum_{j_{k}=1}^{n} \frac{a\left(j_{1}, \ldots, j_{k}\right) \prod_{i=1}^{k}\left(x_{\gamma(i)}^{\left(j_{i}\right)}-x_{\gamma(0)}^{\left(j_{i}\right)}\right)}{\prod_{i=1}^{k}\left|x_{\gamma(i)}-x_{\gamma(0)}\right|^{m+1}},
\end{aligned}
$$


where $H$ is the group of permutations of $\{0,1, \ldots, k\}, a:\{1, \ldots, n\}^{k} \rightarrow \mathbf{R}$, and $x_{l}^{(q)}$ denotes the $q$-th component of $x_{l} \in \mathbf{R}^{n}$. For instance in the case of the standard curvature as in (2), we have that $a(i, j)=0$, if $i \neq j$, and $a(i, i)=1$, for $i \in\{1, \ldots, n\}$.

Definition 1. We will say that $P_{m, k, n} \in \mathcal{P}$ is an $(m, k, n)$-curvature function of the Melnikov type if $0 \leq P_{m, n, k} \not \equiv 0$.

\subsection{Statement and proof of the main theorem.}

Theorem 2. Let $n \geq 2$. If $m \geq 2$, or $k \neq 2$, $(m, n, k)$-curvature functions of the Melnikov type do not exist. For $m=1, k=2$, the $(1,2, n)$-curvature functions defined by (2) are uniquely characterized (up to constant multiples) in the class $\mathcal{P}$ by being invariant under the action of $O(n)$ (where a transformation acts simultaneously on the variables $x_{i}$, for all $i)$.

Proof: Suppose $P_{m, k, n}$ is one such function. The first observation is that regardless of $m$, or $n,(m, k, n)$-curvature functions cannot exist for odd $k$. This is easily seen by applying the transformation $x_{i} \rightarrow-x_{i}$, for all $i$, under which the function changes sign. By translation-invariance which is implicit in the form (5), we can assume that $x_{0}=\mathbf{0}$, and, from now on, think of $P_{m, k, n}$ as a function of $k$, not $k+1$, variables. We also observe that the sum over the permutations can be performed by summing functions $\mathcal{S}_{i}$, where $i=0, \ldots, k$, such that for an element $\sigma$ of $H$, the group of permutations of $\{1, \ldots, k\}$,

$$
\mathcal{S}_{0} \equiv \sum_{\sigma \in H} \sum_{j_{1}=1}^{n} \cdots \sum_{j_{k}=1}^{n} \frac{a\left(j_{1}, \ldots, j_{k}\right) \prod_{i=1}^{k} x_{\sigma(i)}^{\left(j_{i}\right)}}{\prod_{i=1}^{k}\left|x_{i}\right|^{m+1}}
$$

and $\mathcal{S}_{l}$ is obtained from $\mathcal{S}_{0}$ via the transformation $x_{l} \rightarrow-x_{l}$, and $x_{j} \rightarrow x_{j}-x_{l}$, for $j \neq l$. Thus

$$
P_{m, k, n}=\sum_{i=0}^{k} \mathcal{S}_{l}
$$

This decomposition will allow us to understand all the $S_{i}$ 's once we understand $S_{0}$. Now we observe that nonnegativity is an invariance under the action of $O(n)$ on the variables. We can thus integrate (or average) over $O(n)$, and since the denominators in the expressions for the $S_{i}$ 's are invariant, we can replace our original function by one that is of exactly the same form but invariant under the action of $O(n)$. That is, we can 
assume that the coefficients $a\left(j_{1}, \ldots, j_{k}\right)$ are so that the polynomial in the numerator of each $\mathcal{S}_{i}$ is invariant. For convenience, we continue to denote the new functions by $P_{m, k, n}$ and $\mathcal{S}_{i}$ respectively. Notice that with these new functions we can still obtain $P_{m, k, n}$ from the $\mathcal{S}_{i}$ 's by means of (7), and each $\mathcal{S}_{i}$ from $\mathcal{S}_{0}$ as before. In fact, the only change is to the coefficients of the polynomials in the numerators of $\mathcal{S}_{0}$ (note that these are the same coefficients in each $\mathcal{S}_{i}$ ). We therefore wish to study the new polynomial in the numerator of $\mathcal{S}_{0}$. Let us denote this polynomial by $Q_{m, k, n}\left(x_{1}, \ldots, x_{k}\right)$. Using the properties of this polynomial we will find a simpler, more useful form for it. We define

$$
G_{m, k, n}\left(x_{1}, \ldots, x_{k}\right) \equiv \sum_{\sigma \in H} \prod_{i=1}^{\frac{k}{2}} x_{\sigma(2 i-1)} \cdot x_{\sigma(2 i)},
$$

and observe that both $Q_{m, k, n}$ and $G_{m, k, n}$, are invariant (symmetric) under permutations of the variables. Furthermore, the coordinates of any variable $x_{i}$ appear linearly in them. Finally, if we set $x_{i}=x$, for all $i$, then, by homogeneity and rotation-invariance, both of these polynomials must be multiples of $|x|^{k}$. We now invoke

Lemma 3. Suppose $R\left(x_{1}, \ldots, x_{k}\right)$ is a polynomial in the coordinates of $x_{1}, \ldots, x_{k} \in \mathbf{R}^{n}$, such that the coordinates of the variables appear at most linearly (for example $\left(x_{2}^{(1)}\right)^{2}$, and $x_{1}^{(1)} x_{1}^{(2)}$ are not allowed). If $R$ is also symmetric under permutations of the variables $x_{1}, \ldots, x_{k}$, then it is uniquely determined by its restriction to the set $x_{1}=\cdots=x_{k}$.

Proof: Suppose $R$ is one such polynomial, and that $r(x) \equiv R(x, \ldots, x) \equiv$ 0 . We will show that $R \equiv 0$. For this it suffices to show that there is a one-to-one correspondence between the distinct coefficients (i.e. those which are not determined by symmetry) of terms of $R$, and those of $r$. Note that the assumption on $R$ implies that $r$ can have degree at most $k$. Now the generic term in a polynomial of degree at most $k$ is of the form $c(\theta) \prod_{l=1}^{n}\left(x^{(l)}\right)^{\theta(l)}$, where $\theta:\{1, \ldots, n\} \rightarrow\{0,1, \ldots, k\}$, and $r$ is obtained by the sum over all possible $\theta$. Such a term in $r$ must arise exactly from those terms of $R$ which have $l$-th coordinates of exactly $\theta(l)$ of the $k$ variables appearing. By symmetry, all such terms have the same coefficient. We can then group the distinct terms of $R$ in that manner, and then $c(\theta)$ is a positive integer multiple of the appropriate coefficient in $r$, as described above. This establishes the required correspondence. 
From the above lemma we conclude that $Q_{m, k, n}$ is a constant multiple of $G_{m, k, n}$. In particular, this establishes the uniqueness (up to constant multiples) of the $(1,2, n)$-curvature functions in (2) among the ones with the same symmetries as claimed. We will now assume, without loss of generality, that

$$
Q_{m, k, n}=G_{m, k, n}
$$

and we start by showing that, with this substitution, $P_{m, k, n}$ takes positive values (otherwise we would have needed to take $Q_{m, k, n}=-G_{m, k, n}$ ). Let $x, y \in \mathbf{R}^{n} \backslash\{\mathbf{0}\}$ be such that

$$
x \cdot y=0 .
$$

Choose $\lambda \in(0, \infty)$, and set $x_{1}=x$, and $x_{i}=\lambda^{i} y$, for $2 \leq i \leq k$. We study the behavior of $P_{m, k, n}\left(x_{1}, \ldots, x_{k}\right)$ as $\lambda \rightarrow \infty$. First, we observe that

$$
\mathcal{S}_{0}=0,
$$

whereas

$$
\mathcal{S}_{1}=k \sum_{\alpha \in G}\left\{\frac{(-x) \cdot\left(\lambda^{\alpha(2)} y-x\right) \prod_{2}^{\frac{k}{2}}\left(\left(\lambda^{\alpha(2 i-1)} y-x\right) \cdot\left(\lambda^{\alpha(2 i)} y-x\right)\right)}{|x|^{m+1} \prod_{i=2}^{k}\left|\lambda^{i} y-x\right|^{m+1}}\right\},
$$

where $G$ is the group of permutations of $\{2, \ldots, k\}$. An easy computation using (10) shows that

$$
\lambda^{2} \prod_{i=2}^{k}\left(\lambda^{i}\right)^{m} \mathcal{S}_{1}=\frac{k(k-2) !}{|x|^{m-1}|y|^{m(k-1)+1}}\left(1+O\left(\frac{1}{\lambda}\right)\right) .
$$

On the other hand,

$$
\mathcal{S}_{2}=\frac{k !|y|^{k} \lambda^{2} \prod_{i=2}^{k}\left(\lambda^{i}\right)}{\left|x-\lambda^{2} y\right|^{m+1}\left|\lambda^{2} y\right|^{m+1} \prod_{i=3}^{k}\left|\lambda^{i} y-\lambda^{2} y\right|^{m+1}}\left(1+O\left(\frac{1}{\lambda}\right)\right),
$$

so that

$$
\left(\lambda^{2 m}\right)^{2} \prod_{i=3}^{k}\left(\lambda^{i}\right)^{m} \mathcal{S}_{2}=\frac{k !}{|y|^{m k}}\left(1+O\left(\frac{1}{\lambda}\right)\right) .
$$


Similarly, for $i \geq 3$,

$$
\left(\lambda^{i m}\right)^{i} \prod_{j=i+1}^{k}\left(\lambda^{j}\right)^{m}\left|\mathcal{S}_{i}\right| \leq \frac{k !}{|y|^{m k}}\left(1+O\left(\frac{1}{\lambda}\right)\right) .
$$

Note that we needed the exact form of the lowest order term in $\mathcal{S}_{2}$ only for the case $m=1$. Hence, for $\lambda$ large enough, $P_{m, k, n}$ takes positive values. To show that $P_{m, k, n}$ takes negative values, we let $x, y$, be as before, and set $x_{1}=x, x_{2}=-x, x_{i}=\lambda^{i} y$, for $3 \leq i \leq k$. We first eliminate the case $k=2, m>1$ :

In this case

$$
\begin{aligned}
P_{m, k, n} & =\frac{-2|x|^{2}}{\left(|x|^{m+1}\right)^{2}}+\frac{2(-x \cdot(-2 x))}{2^{m+1}\left(|x|^{m+1}\right)^{2}}+\frac{2 x \cdot 2 x}{2^{m+1}\left(|x|^{m+1}\right)^{2}} \\
& =\frac{2}{|x|^{2 m}}\left(-1+2^{-m}+2^{-m}\right)<0 .
\end{aligned}
$$

We should mention that this case was previously known (see the remarks in [MMV, Section 4]). Now we tackle the case $k>2$, and $m>1$ :

Using (10), we compute

$$
\begin{aligned}
\mathcal{S}_{0}= & \frac{-k(k-2) !|x|^{2} \prod_{i=3}^{k}\left(\lambda^{i}|y|\right)}{\left(|x|^{m+1}\right)^{2}\left(\prod_{i=3}^{k} \lambda^{i}|y|\right)^{m+1}} \\
= & \frac{-k(k-2) !}{|x|^{2 m}|y|^{m(k-2)}\left(\prod_{i=3}^{k} \lambda^{i}\right)^{m}},
\end{aligned}
$$

whereas, for $j=1,2$,

$$
\begin{aligned}
\mathcal{S}_{j} & =\left\{\frac{2|x|^{2} k(k-2) ! \prod_{i=3}^{k}\left(\lambda^{i}|y|\right)}{2^{m+1}\left(|x|^{m+1}\right)^{2}\left(\prod_{i=3}^{k} \lambda^{i}|y|\right)^{m+1}}\right\}\left(1+O\left(\frac{1}{\lambda^{6}}\right)\right) \\
& =\left\{\frac{k(k-2) !}{2^{m}|x|^{2 m}|y|^{m(k-2)}\left(\prod_{i=3}^{k} \lambda^{i}\right)^{m}}\right\}\left(1+O\left(\frac{1}{\lambda^{6}}\right)\right) .
\end{aligned}
$$


We will analyze the higher order term in (19) in the next paragraph. On the other hand,

$$
\begin{aligned}
\mathcal{S}_{3} & =\frac{-k !\left(\lambda^{3}\right)^{3}\left(\prod_{i=4}^{k} \lambda^{i}\right)|y|^{k}}{\left(\lambda^{9}|y|^{3} \prod_{i=4}^{k}\left(\lambda^{i}|y|\right)\right)^{m+1}}\left(1+O\left(\frac{1}{\lambda}\right)\right) \\
& =\left(\frac{-k !}{\lambda^{6 m}\left(\prod_{i=3}^{k} \lambda^{i}\right)^{m}|y|^{m k}}\right)\left(1+O\left(\frac{1}{\lambda}\right)\right),
\end{aligned}
$$

and for $j \geq 4$,

$$
\left|\mathcal{S}_{j}\right| \leq \frac{k !}{\left(\lambda^{j^{2}} \prod_{i=j+1}^{k} \lambda^{i}\right)^{m}|y|^{m k}}\left(1+O\left(\frac{1}{\lambda}\right)\right) .
$$

Thus, for $m>1, P_{m, k, n}$ will take negative values as $\lambda \rightarrow \infty$. Finally, for $k \geq 3$, and $m=1$, we will need to compute the next order terms in $\mathcal{S}_{1}$ and $\mathcal{S}_{2}$, since the lowest order terms cancel when added to $\mathcal{S}_{0}$. The estimates for $\mathcal{S}_{1}$, and $\mathcal{S}_{2}$, are identical and come from the term $\left(|x|^{2}+\left|\lambda^{3} y\right|^{2}\right)$ in the denominator. Note that the next order term in the numerator comes from the terms of the same order as $x \cdot\left(\lambda^{3} y-x\right)(2 x) \cdot\left(\lambda^{4} y-x\right) \prod_{i=3}^{\frac{k}{2}}\left(\lambda^{2 i-1} y \cdot \lambda^{2 i} y\right)$, which have a power of $\lambda$ that is less than the first term computed in (19) by 7 . Thus we find that

$(22) \mathcal{S}_{1}+\mathcal{S}_{2}=\left(\frac{k(k-2) !}{|x|^{2}|y|^{k-2}\left(\prod_{i=3}^{k} \lambda^{i}\right)}\right)\left(1-\frac{|x|^{2}}{\lambda^{6}|y|^{2}}\left(1+O\left(\frac{1}{\lambda}\right)\right)\right)$.

Hence, for large enough $\lambda, P_{m, k, n}$ will take negative values.

Remark 4. We mention in passing that for $m>1$, one can replace the substitution $\lambda^{i} y$, by $i \lambda y$ instead. It was the more delicate case $m=1$ that dictated our choice.

This concludes the proof of our theorem. 


\section{Concluding remarks}

In addition to our theorem above, we note that homogeneity makes the change in sign persist at all scales. That is, in every neighborhood $U$ of (say) $x_{0} \in \mathbf{R}^{n}$, we can find two sets $\left\{x_{1}, \ldots, x_{k}, x_{1}^{\prime}, \ldots, x_{k}^{\prime}\right\} \subset U$, so that $P_{m, k, n}$ assumes values of opposite signs on $\left(x_{1}, \ldots, x_{k}\right)$ and $\left(x_{1}^{\prime}, \ldots, x_{k}^{\prime}\right)$. Furthermore, since $P_{m, k, n}$ is continuous on the set $\left\{\left(x_{1}, \ldots, x_{k}\right): x_{i} \neq x_{j}\right.$, for all $i \neq j\}$, the sets on which it assumes either positive or negative values are open in $\mathbf{R}^{n(k+1)}$, and are thus too large to ignore measuretheoretically, perhaps even if we use our knowledge of the geometric properties of totally unrectifiable $m$-sets for instance. Finally, we note (motivated by a question of Tolsa) that although we implicitly assumed that the class $\mathcal{P}$ contained only functions symmetric in the variables $x_{i}$ as the motivating measure-theoretic problem suggests, there is no loss of generality even then, since nonnegativity is an invariance under the action of the permutation group $S_{k+1}$ on the points $x_{0}, \ldots, x_{k}$, and one can replace any expression by a symmetric one. Again the fact that the denominators are symmetric plays a role here.

\section{References}

[Ch] M. Christ, A $T(b)$ theorem with remarks on analytic capacity and the Cauchy integral, Colloq. Math. 60/61 (1990), 1367-1381.

[Da] G. DAvid, Unrectifiable 1-sets have vanishing analytic capacity, Rev. Mat. Iberoamericana 14(2) (1998), 369-479.

[DM] G. David And P. Mattila, Removable sets for Lipschitz harmonic functions in the plane, Preprint, University of Jyväskylä.

[DS1] G. DAVID And S. Semmes, Singular Integrals and Rectifiable Sets in $R^{n}$, Au-dela des graphes Lipschitziens, Astérisque 193 (1991).

[DS2] G. DAvid And S. Semmes, Analysis of and on Uniformly Rectifiable Sets, Math. Surveys Monographs 38, Amer. Math. Soc. (1993).

[Jo] P. W. Jones, Rectifiable sets and the traveling salesman problem, Invent. Math. 102 (1990), 1-15.

[MMV] P. Mattila, M. S. Melnikov and J. Verdera, The Cauchy integral, analytic capacity, and uniform rectifiability, Ann. of Math. (2) 144 (1996), 127-136.

[Ma] P. Mattila, Singular integrals, analytic capacity and rectifiability, J. Four. Anal. Appl. 3, special issue (1997), 797-812. 
[Me] M. S. Melnikov, Analytic capacity: discrete approach and curvature of measure, Sb. Math. 186(6) (1995), 827-846.

[MP] P. Mattila and P. V. Paramonov, On geometric properties

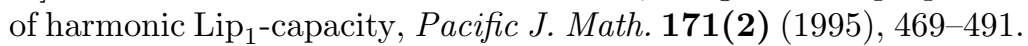

[MV] M. Melnikov And J. Verdera, A geometric proof of the $L^{2}$ boundedness of the Cauchy integral on Lipschitz graphs, Internat. Math. Res. Notices 7 (1995), 325-331.

\author{
Department of Mathematics \\ Rice University \\ Houston, TX 77005 \\ U.S.A. \\ e-mail: farag@math.rice.edu
}

Primera versió rebuda el 17 de setembre de 1998, darrera versió rebuda el 10 de novembre de 1998 